\title{
Characterizations of Certain Recently Introduced Discrete Distributions
}

\author{
G.G. Hamedani
}

Correspondence: G.G. Hamedani, Department of Mathematics, Statistics and Computer Science, Marquette University Milwaukee, WI 53201-1881, USA.

Received: September 19, 2018

doi:10.5539/ijsp.v8n1p56
Accepted: November 8, 2018 Online Published: December 9, 2018

URL: https://doi.org/10.5539/ijsp.v8n1p56

\begin{abstract}
Characterizations of certain recently introduced discrete distributions are presented to complete, in some way, the works cited in the References.
\end{abstract}

Keywords: discrete distributions, generalized discrete distributions, characterizations

\section{Introduction}

The problem of characterizing a distribution is an important problem in applied sciences, where an investigator is vitally interested to know if their model follows the right distribution. To this end, the investigator relies on conditions under which their model would in fact follow specically the chosen distribution. Para and Jan (2018) introduced a new discrete probability model via compounding two-parameter discrete inverse Weibull distribution with the beta distribution of the rst kind, called Discrete Inverse Weibull Beta (DIWB) distribution. They argue that "such a distribution is needed in medical science and other related fields to fit various discrete data sets". Bhati and Bakouch (2018) proposed a discrete distribution called New Geometric Discrete Pareto (NGDP) distribution whose hazard function, among other things, is infinitely divisible. Hussain et al.introduced a discrete distribution called Two Parameter Discrete Lindley (TPDL), which they believe " has the least loss of information when applied to a number of data sets ( in an over and under dispersed structure)". Grine and Zeghdoudi (2017) proposed "a recent version of the compound Poisson distribution" called Poisson Quasi Lindley (PQL) distribution by "compounding Poisson and quasi Lindley distributions". Kumar and Sreejakumari (2016) developed "an extended version of the modified geometric distribution" called Extended Inverted Geometric (EIG) distribution and investigated some of its properties. Jayakumar and Sankaran (2018) proposed a generalization of Weibull distribution based on an arbitrary baseline cumulative distribution function $G(x)$, called (DG) distribution and investigated some of its properties. Prasanth and Sandhya (2016) introduced a generalization of discrete uniform distribution called Harris Discrete Uniform (HDU) distribution. Prasanth and Sandhya (2016) proposed a generalization of discrete uniform distribution called Harris Discrete Uniform (HDU) distribution. Supanekar and Shirke (2015) introduced a new discrete family of distributions called Discrete Family (DF). In this paper, we present three characterizations of these distributions based on: (i) conditional expectation of certain function of the random variable; (ii) the hazard rate function and (iii) the reverse hazard rate function. It should be added that the content of this work theoretical and we leave its applications to the applied scientists. The cumulative distribution function (cdf), $F(x)$, corresponding probability mass function (pmf), $f(x)$, hazard rate function, $h_{F}(x)$, and reverse hazard rate function, $r_{F}(x)$, of DIWB are given, respectively, by

$$
\begin{gathered}
F(x)=F(x ; \alpha, \beta, \gamma)=\frac{1}{B(\alpha, \beta)} B\left(\beta,(x+1)^{-\gamma}+\alpha\right), \\
f(x)=f(x ; \alpha, \beta, \gamma)=\left\{\begin{array}{ll}
\frac{\alpha+1}{\alpha+\beta+1}, & x \in N^{*}, \\
\frac{B\left(\beta,(x+1)^{-\gamma}+\alpha\right)-B\left(\beta,(x)^{-\gamma}+\alpha\right)}{B(\alpha, \beta)}, & x \in N
\end{array},\right. \\
h_{F}(x)=h_{F}(x ; \alpha, \beta, \gamma)=\left\{\begin{array}{ll}
\frac{\alpha+1}{\alpha+\beta+1}, & x=0 \\
\frac{B\left(\beta,(x+1)^{-\gamma}+\alpha\right)-B\left(\beta,(x)^{-\gamma}+\alpha\right)}{B(\alpha, \beta)-B\left(\beta,(x+1)^{-\gamma}+\alpha\right)}, & x \in N
\end{array},\right.
\end{gathered}
$$




$$
r_{F}(x)=r_{F}(x ; \alpha, \beta, \gamma)=\left\{\begin{array}{ll}
1, & x=0 \\
1-\frac{B\left(\beta,(x)^{-\gamma}+\alpha\right)}{B\left(\beta,(x+1)^{-\gamma}+\alpha\right)}, & x \in N
\end{array},\right.
$$

where $\alpha, \beta, \gamma$ are all positive parameters, $\mathbb{N}^{*}=\{0\} \cup \mathbb{N}(\mathbb{N}$ is the set of all positive integers $)$ and

$$
B\left(\beta,(x+1)^{-\gamma}+\alpha\right)=\frac{\Gamma(\beta) \Gamma\left((x+1)^{-\gamma}+\alpha\right)}{\Gamma\left(\beta+(x+1)^{-\gamma}+\alpha\right)} .
$$

The cdf , pmf, hazard rate function and reverse hazard rate function of NGPD are given, respectively, by

$$
\begin{gathered}
F(x)=F(x ; \alpha, q)=1-\frac{q^{x+1}}{(x+2)^{\alpha}}, \quad x \in \mathbb{N}^{*}, \\
F(x)=F(x ; \alpha, q)=\frac{q^{x}}{(x+2)^{\alpha}}-\frac{q^{x+1}}{(x+2)^{\alpha}}, \quad x \in \mathbb{N}^{*}, \\
h_{F}(x)=h_{F}(x ; \alpha, q)=1-\frac{1}{q}\left(\frac{x+2}{x+1}\right)^{\alpha}, \quad x \in \mathbb{N}^{*}, \\
r_{F}(x)=r_{F}(x ; \alpha, q)=1-\frac{(x+1)^{\alpha}}{1-\frac{q^{x}+1}{(x+2)^{\alpha}}}, \quad x \in \mathbb{N}^{*},
\end{gathered}
$$

where $\alpha>0, q \in(0,1]$ are parameters.

The cdf, pmf and hazard rate function and reverse hazard function of TPDL are given, respectively, by

$$
\begin{gathered}
F(x)=F(x ; \beta, p)=1-\frac{[(1-p)(1+\beta x)+\beta] p^{x+1}}{1+p(1-\beta)}, \quad x \in \mathbb{N}^{*}, \\
f(x)=f(x ; \beta, p)=\frac{(1-p)^{2}(1+\beta x) p^{x}}{1+p(1-\beta)}, \quad x \in \mathbb{N}^{*}, \\
h_{F}(x)=h_{F}(x ; \beta, p)=\frac{(1-p)^{2}(1+\beta x)}{p[(1-p)(1+\beta x)+\beta]}, \quad x \in \mathbb{N}^{*}, \\
r_{F}(x)=r_{F}(x ; \beta, p)=\frac{(1-p)^{2}(1+\beta x) p^{x}}{1+p(\beta-1)-p[(1-p)(1+\beta x)+\beta] p^{x+1}}, \quad x \in \mathbb{N}^{*},
\end{gathered}
$$

where $\beta>0, p \in(0,1]$ are parameters.

The cdf, pmf, hazard rate function and reverse hazard function of PQL are given, respectively, by

$$
\begin{gathered}
F(x)=F(x ; \alpha, \theta)=1-\frac{\alpha+2 \theta+\alpha \theta+\theta x+1}{(1+\alpha)(1+\theta)^{x+2}}, \quad x \in \mathbb{N}^{*}, \\
f(x)=f(x ; \alpha, \theta)=\frac{\theta(\alpha+\theta+\alpha \theta+\theta x)}{(1+\alpha)(1+\theta)^{x+2}}, \quad x \in \mathbb{N}^{*}, \\
h_{F}(x)=h_{F}(x ; \alpha, \theta)=\frac{\theta(\alpha+\theta+\alpha \theta+\theta x)}{\alpha+2 \theta+\alpha \theta+\theta x+1}, \quad x \in \mathbb{N}^{*}, \\
r_{F}(x)=r_{F}(x ; \alpha, \theta)=\frac{\theta(\alpha+\theta+\alpha \theta+\theta x)}{(1+\alpha)(1+\theta)^{x+2}-(\alpha+2 \theta+\alpha \theta+\theta x+1)}, \quad x \in \mathbb{N}^{*},
\end{gathered}
$$

where $\alpha>-1$ and $\theta>0$ are parameters.

The cdf, pmf and hazard rate function and reverse hazard function of EIG are given, respectively, by

$$
F(x)=F\left(x ; p_{1}, \theta\right)=1-\frac{\theta^{x}\left[\left(1-p_{1} \theta\right)-(1-\theta) p_{1}^{x+1}\right]}{1-p_{1}}, \quad x \in \mathbb{N}^{*},
$$




$$
\begin{gathered}
f(x)=f\left(x ; p_{1}, \theta\right)=\frac{(1-\theta)\left(1-p_{1} \theta\right)\left(1-p_{1}^{x}\right) \theta^{x-1}}{1-p_{1}}, \quad x \in \mathbb{N}^{*}, \\
h_{F}(x)=h_{F}\left(x ; p_{1}, \theta\right)=\frac{(1-\theta)\left(1-p_{1} \theta\right)\left(1-p_{1}^{x}\right)}{\theta\left[\left(1-p_{1} \theta\right)-(1-\theta) p_{1}^{x+1}\right]}, \quad x \in \mathbb{N}^{*}, \\
r_{F}(x)=r_{F}\left(x ; p_{1}, \theta\right)=\frac{(1-\theta)\left(1-p_{1} \theta\right)\left(1-p_{1}^{x}\right) \theta^{x-1}}{\left(1-p_{1}\right)-\theta^{x}\left[\left(1-p_{1} \theta\right)-(1-\theta) p_{1}^{x+1}\right]}, \quad x \in \mathbb{N}^{*},
\end{gathered}
$$

where $\theta \in(0,1)$ and $0<p_{1} \neq 1$ are parameters such that $p_{1} \theta<1$.

The cdf, pmf, hazard rate function and reverse hazard function of DG are given, respectively, by

$$
\begin{gathered}
F(x)=F(x ; \alpha, \theta)=\frac{G^{\alpha}(x+1)}{\theta+\bar{\theta} G^{\alpha}(x)}, \quad x \in \mathbb{N}^{*}, \\
f(x)=f(x ; \alpha, \theta)=\frac{\theta}{1-\theta}\left[\frac{1}{\theta+\bar{\theta} G^{\alpha}(x)}-\frac{1}{\theta+\bar{\theta} G^{\alpha+1}(x)}\right], \quad x \in \mathbb{N}^{*}, \\
h_{F}(x)=h_{F}(x ; \alpha, \theta)=\frac{G^{\alpha}(x+1)-G^{\alpha}(x)}{\left(1+G^{\alpha}(x+1)\right)\left(\theta+\bar{\theta} G^{\alpha}(x)\right)}, \quad x \in \mathbb{N}^{*}, \\
r_{F}(x)=r_{F}(x ; \alpha, \theta)=\frac{\theta\left[G^{\alpha}(x+1)-G^{\alpha}(x)\right]}{G^{\alpha}(x+1)\left(\theta+\bar{\theta} G^{\alpha}(x)\right)}, \quad x \in \mathbb{N}^{*},
\end{gathered}
$$

where $\alpha$ and $\theta$ are positive parameters, $\bar{\theta}=1-\theta$ and $G(x)$ is a baseline cdf with $G(0)=0$. The cdf, pmf and hazard rate function of HDU are given, respectively, by

$$
\begin{gathered}
F(x)=F(x ; \alpha, \theta, p)=1-\frac{\theta^{1 / p}(\alpha-x)}{\left[\alpha^{p}-(1-\theta)(\alpha-x)^{p}\right]^{1 / p}}, \quad x \in I=\{1,2, \ldots, \alpha\}, \\
f(x)=f(x ; \alpha, \theta, p)=\theta^{1 / p}\left[\frac{\alpha-x+1}{\left[\alpha^{p}-(1-\theta)(\alpha-x+1)^{p}\right]^{1 / p}}-\frac{\alpha-x}{\left[\alpha^{p}-(1-\theta)(\alpha-x)^{p}\right]^{1 / p}}\right], \quad x \in I, \\
h_{F}(x)=h_{F}(x ; \alpha, \theta, p)=\theta^{1 / p}\left[\frac{(\alpha-x+1)\left[\alpha^{p}-(1-\theta)(\alpha-x)^{p}\right]^{1 / p}}{(\alpha-x)\left[\alpha^{p}-(1-\theta)(\alpha-x+1)^{p}\right]^{1 / p}}-1\right], \quad x \in I,
\end{gathered}
$$

where $\alpha \in \mathbb{N}, \theta>0$ and $p \in \mathbb{N}$ are parameters.

The cdf, pmf and hazard rate function of DF are given, respectively, by

$$
\begin{gathered}
F(x)=F(x ; \alpha, \sigma)=\frac{1-\bar{G}(x+1 ; \sigma)}{1-\bar{\alpha} G(x+1 ; \sigma)}, \quad x \in \mathbb{N}^{*}, \\
f(x)=f(x ; \alpha, \sigma)=\frac{\alpha}{\bar{\alpha}}\left\{\frac{1}{1-\bar{\alpha} \bar{G}(x ; \sigma)}-\frac{1}{1-\bar{\alpha} \bar{G}(x+1 ; \sigma)}\right\}, \\
h_{F}(x)=h_{F}(x ; \alpha, \sigma)=\frac{\bar{G}(x ; \sigma)-\bar{G}(x+1 ; \sigma)}{\bar{G}(x+1 ; \sigma)-(1-\bar{\alpha} \bar{G}(x ; \sigma))}, \quad x \in \mathbb{N}^{*}, \\
r_{F}(x)=r_{F}(x ; \alpha, \sigma)=\frac{\bar{\alpha}[\bar{G}(x ; \sigma)-\bar{G}(x+1 ; \sigma)]}{(1-\bar{G}(x+1 ; \sigma))-(1-\bar{\alpha} \bar{G}(x ; \sigma))}, \quad x \in \mathbb{N}^{*},
\end{gathered}
$$

where $\alpha>0, \theta>0$ are parameters and $G(x)$ is a continuous baseline cdf. 
Remarks 1. (a) In defining the pmf of DIWB, Para and Jan did not make the distinction we have made here in (2) for $x=$ $O$ and $x \in \mathbb{N}$. (b) Bahti and Bakouch, Hussain et al. and Supanekar and Shirke, have defined their survival functions as $F(x)=1-F(x-1)$ (c) Without loss of generality, we assume $=0$ for the PQL distribution. (d) Although Jayakumar and Sankaran defined the survival function as $P(X>x)$, but they actually use $P(X \geq x)$ as their survival function when they obtain the hazard and reverse hazard functions. We also believe that they assume $G(0)=0$. (e) The hazard rate function given on page 85 of Supanekar and Shirke is incorrect; the correct formula is (30) given above.

\section{Characterization Results}

We present our characterizations (i) (iii) via three subsections 2.1, 2.2 and 2.3.

2.1 Characterizations in Terms of the Conditional Expectation of Certain Function of the Random Variable

Proposition 2.1.1. Let $X: \Omega \rightarrow \mathbb{N}^{*}$ be a random variable. The pmf of $X$ is (2) if and only if

$$
E\left\{B\left(\beta,(X+1)^{-\gamma}+\alpha\right)+B\left(\beta,(X)^{-\gamma}+\alpha\right) \mid X>k\right\}=\mathrm{B}(\alpha, \beta)+B\left(\beta,(k+1)^{-\gamma}+\alpha\right) .
$$

Proof. If $\mathrm{X}$ has pmf (2), then the left-hand side of (32) will be

$$
\begin{gathered}
\frac{(1-F(k))^{-1}}{B(\alpha, \beta)} \sum_{x=k+1}^{\infty}\left[B^{2}\left(\beta,(x+1)^{-\gamma}+\alpha\right)+B^{2}\left(\beta,(x)^{-\gamma}+\alpha\right)\right]=\frac{(1-F(k))^{-1}}{B(\alpha, \beta)}\left[B^{2}(\alpha, \beta)+B^{2}\left(\beta,(k+1)^{-\gamma}+\alpha\right)\right] \\
=\frac{1}{B(\alpha, \beta)-B\left(\beta,(k+1)^{-\gamma}+\alpha\right)}\left[B^{2}(\alpha, \beta)+B^{2}\left(\beta,(k+1)^{-\gamma}+\alpha\right)\right] \\
=B(\alpha, \beta)-B\left(\beta,(k+1)^{-\gamma}+\alpha\right) .
\end{gathered}
$$

Conversely, if (32) holds, then

$$
\begin{aligned}
\sum_{x=k+1}^{\infty}\left[\left\{B\left(\beta,(x+1)^{-\gamma}+\alpha\right)\right.\right. & \left.\left.+B^{2}\left(\beta,(x)^{-\gamma}+\alpha\right)\right\} f(x)\right]=(1-F(k))\left[B(\alpha, \beta)+B\left(\beta,(k+1)^{-\gamma}+\alpha\right)\right] \\
= & \{(1-F(k))-f(k+1)\}\left[B(\alpha, \beta)+B\left(\beta,(k+1)^{-\gamma}+\alpha\right)\right] .
\end{aligned}
$$

From (32), we also have

$$
\begin{aligned}
& \frac{(1-F(k))^{-1}}{B(\alpha, \beta)} \sum_{x=k+2}^{\infty}\left[\left\{B\left(\beta,(x+1)^{-\gamma}+\alpha\right)+B\left(\beta,(x)^{-\gamma}+\alpha\right)\right\} f(x)\right] \\
= & (1-F(k+1))^{-1}\left[B\left(\beta,(k+1)^{-\gamma}+\alpha\right)+B\left(\beta,(k+2)^{-\gamma}+\alpha\right)\right] .
\end{aligned}
$$

Now, subtracting (34) from (33), we arrive at

$$
(1-F(k+1))\left\{B\left(\beta,(k+1)^{-\gamma}+\alpha\right)-B\left(\beta,(k+2)^{-\gamma}+\alpha\right)\right\}=\left\{B\left(\beta,(k+2)^{-\gamma}+\alpha\right)-B(\alpha, \beta)\right\} f(k+1) .
$$

From the last equality, we have

$$
h_{F}(k+1)=\frac{f(k+1)}{(1-F(k+1))}=\frac{B\left(\beta,(k+1)^{-\gamma}+\alpha\right)-B\left(\beta,(k+2)^{-\gamma}+\alpha\right)}{B\left(\beta,(k+2)^{-\gamma}+\alpha\right)-B(\alpha, \beta)},
$$

which, in view of (3), implies that $X$ has pmf (2).

Proposition 2.1.2. Let $X: \Omega \rightarrow \mathbb{N}^{*}$ be a random variable. The pmf of $X$ is (6) if and only if

$$
E=\left[\left\{\frac{q^{X}}{(X+1)^{\alpha}}-\frac{q^{X+1}}{(X+2)^{\alpha}}\right\} \mid X>k\right]=\frac{q^{k+1}}{(k+2)^{\alpha}} .
$$

Proof. The proof is similar to that of Proposition 2.1.1. We, however, give the proof for the sake of completeness. If $X$ has pmf (6), then the left-hand side of (35) will be

$$
(1-F(k))^{-1}=\sum_{x=k+1}^{\infty}\left[\left\{\left(\frac{q^{x}}{(x+1)^{\alpha}}\right)^{2}-\left(\frac{q^{x+1}}{(x+2)^{\alpha}}\right)^{2}\right\} \mid X>k\right]=\frac{(k+2)^{\alpha}}{q^{k+1}}\left(\frac{q^{k+1}}{(k+2)^{\alpha}}\right)^{2}=\frac{q^{k+1}}{(k+2)^{\alpha}} .
$$


Conversely, if (35) holds, then

$$
\sum_{x=k+1}^{\infty}\left[\left\{\left(\frac{q^{x}}{(x+1)^{\alpha}}\right)^{2}-\left(\frac{q^{x+1}}{(x+2)^{\alpha}}\right)^{2}\right\} f(x)\right]=(1-F(k))\left(\frac{q^{k+1}}{(k+2)^{\alpha}}\right)=[(1-F(k+1))+f(k+1)]\left(\frac{q^{k+1}}{(k+2)^{\alpha}}\right) .
$$

From (35), we also have

$$
\sum_{x=k+2}^{\infty}\left[\left\{\left(\frac{q^{x}}{(x+1)^{\alpha}}\right)^{2}-\left(\frac{q^{x+1}}{(x+2)^{\alpha}}\right)^{2}\right\} f(x)\right]=(1-F(k+1))\left(\frac{q^{k+2}}{(k+3)^{\alpha}}\right) .
$$

Now, subtracting (37) from (36), we arrive at

$$
(1-F(k+1))=\left\{\frac{q^{k+1}}{(k+2)^{\alpha}}-\frac{q^{k+2}}{(k+2)^{\alpha}}\right\}=\left(\frac{q^{k+1}}{(k+2)^{\alpha}}\right) f(k+1) .
$$

which, in view of (7), implies that $X$ has pmf (6).

Proposition 2.1.3. Let $X: \Omega \rightarrow \mathbb{N}^{*}$ be a random variable. The pmf of $X$ is (10) if and only if

$$
E\left[\left(\frac{1}{(1+\beta X)}\right) \mid X>k\right]=\frac{(1-p)}{(1-p)(1+\beta k)+\beta} .
$$

Proof. If $X$ has pmf (10), then the left-hand side of (38) will be

$$
(1-F(k))^{-1} \sum_{x=k+1}^{\infty}\left\{\frac{(1-p)^{2} p^{x}}{1+p(\beta-1)+\beta}\right\}=\frac{1+p(\beta-1)}{[(1-p)(1+\beta k)+\beta] p^{k+1}}\left(\frac{(1-p) p^{k+1}}{1+p(\beta-1)}\right)=\frac{(1-p)}{(1-p)(1+\beta k)+\beta} .
$$

Conversely, if (38) holds, then

$$
\sum_{x=k+1}^{\infty}\left\{\left(\frac{1}{(1+\beta x)}\right) f(x)\right\}=(1-F(k))\left(\frac{(1-p)}{(1-p)(1+\beta k)+\beta}\right)=[(1-F(k+1))+f(k+1)]\left(\frac{(1-p)}{(1-p)(1+\beta k)+\beta}\right) .
$$

From (38), we also have

$$
\sum_{x=k+2}^{\infty}\left\{\left(\frac{1}{(1+\beta x)}\right) f(x)\right\}=(1-F(k+1))\left(\frac{(1-p)}{(1-p)(1+\beta(k+1))+\beta}\right) .
$$

Now, subtracting (40) from (39), we arrive at

$$
\begin{aligned}
& \left(\frac{1}{1+\beta(k+1)}-\frac{(1-p)}{(1-p)(1+\beta k)+\beta}\right) f(k+1)=(1-F(k+1))\left(\frac{(1-p)}{(1-p)(1+\beta k)+\beta}-\frac{(1-p)}{(1-p)(1+\beta(k+1))+\beta}\right), \\
& \left(\frac{\beta p}{(1+\beta(k+1))[(1-p)(1+\beta k)+\beta]}\right) f(k+1)=(1-F(k+1))\left(\frac{\beta(1-p)^{2}}{[(1-p)(1+\beta k)+\beta][(1-p)(1+\beta(k+1))+\beta]}\right),
\end{aligned}
$$

or

$$
\frac{f(k+1)}{(1-F(k+1))}\left(\frac{(1-p)^{2}(1+\beta(k+1))}{p((1-p)(1+\beta(k+1))+\beta)}\right),
$$

which, in view of (11), implies that $\mathrm{X}$ has pmf (10).

Proposition 2.1.4. Let $X: \Omega \rightarrow \mathbb{N}^{*}$ be a random variable. The pmf of $X$ is (14) if and only if

$$
E\left[\left(\frac{1}{(1+X)}\right) \mid X>k\right]=\frac{\theta}{\theta(2+k)+1} \text {. }
$$

Proof. If $X$ has pmf (14), then the left-hand side of (41) will be

$$
(1-F(x))^{-1}=\sum_{x=k+1}^{\infty} \frac{\theta^{2}}{(1+\theta)^{x+2}}=\frac{(1+\theta)^{k+2}}{\theta(2+k)+1}\left(\frac{\theta^{2}}{(1+\theta)^{k+2}}\right)=\frac{\theta}{\theta(2+k)+1} .
$$

Conversely, if (41) holds, then 


$$
\sum_{x=k+1}^{\infty}\left\{\left(\frac{1}{(1+x)}\right) f(x)\right\}=(1-F(k))\left(\frac{\theta}{\theta(2+k)+1}\right)=[(1-F(k+1)) f(k+1)]\left(\frac{\theta}{\theta(2+k)+1}\right)
$$

From (41), we also have

$$
\sum_{x=k+2}^{\infty}\left\{\left(\frac{1}{(1+x)}\right) f(x)\right\}=(1-F(k+1))\left(\frac{\theta}{\theta(3+k)+1}\right) .
$$

Now, subtracting (43) from (42), we arrive at

$$
\left(\frac{1}{1+(k+1)}-\frac{\theta}{\theta(2+k)+1}\right) f(k+1)=[(1-F(k+1))]\left(\frac{\theta}{\theta(2+k)+1}-\frac{\theta}{\theta(3+k)+1}\right),
$$

or

$$
\left(\frac{1}{(k+2)(\theta(2+k)+1)}\right) f(k+1)=[(1-F(k+1))]\left(\frac{\theta^{2}}{(\theta(2+k)+1)(\theta(3+k)+1)}\right),
$$

or

$$
\frac{f(k+1)}{1-F(k+1)}=\frac{\theta^{2}(2+k)}{\theta(3+k)+1},
$$

which, in view of (15), implies that $X$ has pmf (14)

Proposition 2.1.5. Let $X: \Omega \rightarrow \mathbb{N}^{*}$ be a random variable. The pmf of $X$ is (18) if and only if

$$
E\left[\left(\frac{1}{\left(1+p_{1}^{X}\right)}\right) \mid X>k\right]=\frac{1-p_{1} \theta}{\left(1-p_{1} \theta\right)-(1-\theta) p_{1}^{k+1}} .
$$

Proof. If $X$ has pmf (18), then the left-hand side of (44) will be

$$
(1-F(k))^{-1} \sum_{x=k+1}^{\infty} \frac{(1-\theta)\left(1-p_{1} \theta\right)}{1-p_{1}} \theta^{x-1}=\frac{1-p_{1}}{\theta^{k}\left(1-p_{1} \theta\right)-(1-\theta) p_{1}^{k+1}}\left(\frac{(1-\theta)\left(1-p_{1} \theta\right)}{(1-\theta)\left(1-p_{1}\right)} \theta^{k}\right)=\frac{1-p_{1} \theta}{\left(1-p_{1} \theta\right)-(1-\theta) p_{1}^{k+1}} .
$$

Conversely, if (44) holds, then

$$
\sum_{x=k+1}^{\infty}\left\{\left(\frac{1}{\left(1+p_{1}^{x}\right)}\right) f(x)\right\}=(1-F(k))\left(\frac{1-p_{1} \theta}{\left(1-p_{1} \theta\right)-(1-\theta) p_{1}^{k+1}}\right)=[(1-F(k+1)) f(k+1)]\left(\frac{1-p_{1} \theta}{\left(1-p_{1} \theta\right)-(1-\theta) p_{1}^{k+1}}\right) .
$$

From (44), we also have

$$
\sum_{x=k+2}^{\infty}\left\{\left(\frac{1}{\left(1+p_{1}^{x}\right)}\right) f(x)\right\}=(1-F(k+1))\left(\frac{1-p_{1} \theta}{\left(1-p_{1} \theta\right)-(1-\theta) p_{1}^{k+2}}\right) .
$$

Now, subtracting (46) from (45), we arrive at

$$
\left(\frac{1}{1-p_{1}^{k+1}}-\frac{1-p_{1} \theta}{\left(1-p_{1} \theta\right)-(1-\theta) p_{1}^{k+1}}\right) f(k+1)=(1-F(k+1))\left(\frac{1-p_{1} \theta}{\left(1-p_{1} \theta\right)-(1-\theta) p_{1}^{k+1}}-\frac{1-p_{1} \theta}{\left(1-p_{1} \theta\right)-(1-\theta) p_{1}^{k+2}}\right),
$$

or

$$
\left(\frac{\theta\left(1-p_{1}\right) p_{1}^{k+1}}{\left(1-p_{1}^{k+1}\right)\left[\left(1-p_{1} \theta\right)-(1-\theta) p_{1}^{k+1}\right]}\right) f(k+1)=(1-F(k+1))\left(\frac{\left(1-p_{1}\right)\left(1-p_{1} \theta\right)(1-\theta) p_{1}^{k+1}}{\left[\left(1-p_{1} \theta\right)-(1-\theta) p_{1}^{k+1}\right]\left[\left(1-p_{1} \theta\right)-(1-\theta) p_{1}^{k+2}\right]}\right),
$$

or

$$
\frac{f(k+1)}{(1-F(k+1))}=\frac{\left(1-p_{1} \theta\right)(1-\theta) p_{1}^{k+1}}{\theta\left[\left(1-p_{1} \theta\right)-(1-\theta) p_{1}^{k+2}\right]},
$$

which, in view of (19), implies that $X$ has pmf (18).

Proposition 2.1.6. Let $X: \Omega \rightarrow \mathbb{N}^{*}$ be a random variable. The pmf of $X$ is (22) if and only if

$$
E\left[\left(\frac{1}{\theta+\bar{\theta} G^{\alpha}(X)}+\frac{1}{\theta+\bar{\theta} G^{\alpha}(X+1)}\right) \mid X>k\right]=1-\frac{1}{\theta+\bar{\theta} G^{\alpha}(k+1)} .
$$


Proof. If X has pmf (22), then the left-hand side of (47) will be

$$
\begin{aligned}
(1-F(k))^{-1} \sum_{x=k+1}^{\infty} \frac{\theta}{1-\theta}\left(\frac{1}{\theta+\bar{\theta} G^{\alpha}(x)^{2}}-\frac{1}{\theta+\bar{\theta} G^{\alpha}(x+1)^{2}}\right) & =\frac{\theta}{1-\theta}\left(\frac{\theta+\bar{\theta} G^{\alpha}(k+1)^{2}}{\theta-\bar{\theta} G^{\alpha}(k+1)^{2}}\right)\left(\frac{1}{\theta+\bar{\theta} G^{\alpha}(k+1)^{2}}-1\right) \\
& =1+\frac{1}{\theta+\bar{\theta} G^{\alpha}(k+1)} .
\end{aligned}
$$

Conversely, if (47) holds, then

$$
\begin{aligned}
(1-F(k))^{-1}\left[\left(\frac{1}{\theta+\bar{\theta} G^{\alpha}(x)^{2}}-\frac{1}{\theta+\bar{\theta} G^{\alpha}(x+1)^{2}}\right) f(x)\right] & =(1-F(k))\left(1+\frac{1}{\theta+\bar{\theta} G^{\alpha}(k+1)^{2}}\right) \\
& =[(1-F(k)) f(k+1)]\left(1+\frac{1}{\theta+\bar{\theta} G^{\alpha}(k+1)^{2}}\right)
\end{aligned}
$$

From (47), we also have

$$
\begin{aligned}
& {\left[\frac{1}{\theta+\bar{\theta} G^{\alpha}(k+1)}-\frac{1}{\theta+\bar{\theta} G^{\alpha}(k+2)}-\left(1+\frac{1}{\theta+\bar{\theta} G^{\alpha}(k+1)}\right)\right] f(k+1)} \\
& =(1-F(k+1))\left[\left(1+\frac{1}{\theta+\bar{\theta} G^{\alpha}(k+1)}\right)-\left(1+\frac{1}{\theta+\bar{\theta} G^{\alpha}(k+2)}\right)\right],
\end{aligned}
$$

or

$$
\left(\frac{1}{\theta+\bar{\theta} G^{\alpha}(k+2)}-1\right) f(k+1)=(1-F(k+1))\left[\frac{\bar{\theta}\left\{G^{\alpha}(k+2)-G^{\alpha}(k+1)\right\}}{\theta+\bar{\theta} G^{\alpha}(k+1)-\theta+\bar{\theta} G^{\alpha}(k+2)}\right]
$$

or

$$
\frac{f(k+1)}{(1-F(k+1))}=\frac{G^{\alpha}(k+2)-G^{\alpha}(k+1)}{\theta+\bar{\theta} G^{\alpha}(k+1)-\theta+\bar{\theta} G^{\alpha}(k+2)},
$$

which, in view of (23), implies that $X$ has pmf (22).

Proposition 2.1.7. Let $X: \Omega \rightarrow I \backslash\{0\}$ be a random variable. The pmf of $X$ is (26) if and only if

$$
E\left[\left(\frac{\alpha-X+1}{\left(\alpha^{p}-(1-\theta)(\alpha-X+1)^{p}\right)^{1 / p}}+\frac{\alpha-X}{\left(\alpha^{p}-(1-\theta)(\alpha-X)^{p}\right)^{1 / p}}\right) \mid X>k\right]=\frac{\alpha-k}{\left(\alpha^{p}-(1-\theta)(\alpha-k)^{p}\right)^{1 / p}} .
$$

Proof. If $X$ has pmf (26), then the left-hand side of (50) will be

$$
\begin{gathered}
(1-F(x))^{-1} \sum_{x=k+1}^{\infty} \alpha^{1 / p}\left(\frac{(\alpha-x+1)^{2}}{\left(\alpha^{p}-(1-\theta)(\alpha-x+1)^{p}\right)^{2 / p}}+\frac{(\alpha-x)^{2}}{\left(\alpha^{p}-(1-\theta)(\alpha-x)^{p}\right)^{2 / p}}\right) \\
=\frac{\left(\alpha^{p}-(1-\theta)(\alpha-x)^{p}\right)^{1 / p}}{(\alpha-k)}\left\{\frac{(\alpha-k)^{2}}{\left(\alpha^{p}-(1-\theta)(\alpha-x)^{p}\right)^{2 / p}}\right\}=\frac{\alpha-k}{\left(\alpha^{p}-(1-\theta)(\alpha-x)^{p}\right)^{1 / p}} .
\end{gathered}
$$

Conversely, if (50) holds, then

$$
\sum_{x=k+1}^{\infty}\left\{\left(\frac{\alpha-x+1}{\left(\alpha^{p}-(1-\theta)(\alpha-x+1)^{p}\right)^{1 / p}}+\frac{\alpha-x}{\left(\alpha^{p}-(1-\theta)(\alpha-x)^{p}\right)^{1 / p}}\right) f(x)\right\}=(1-F(k))\left(\frac{\alpha-k}{\left(\alpha^{p}-(1-\theta)(\alpha-k)^{p}\right)^{1 / p}}\right)=[(1-F(k+1)) f(k+1)] \frac{\alpha-k}{\left(\alpha^{p}-(1-\theta)(\alpha-k)^{p}\right)^{1 / p}} .
$$

From (50), we also have

$$
\sum_{x=k+2}^{\infty}\left\{\left(\frac{\alpha-x+1}{\left(\alpha^{p}-(1-\theta)(\alpha-x+1)^{p}\right)^{1 / p}}+\frac{\alpha-x}{\left(\alpha^{p}-(1-\theta)(\alpha-x)^{p}\right)^{1 / p}}\right) f(x)\right\}=[1-F(k+1)]\left(\frac{\alpha-k-1}{\left(\alpha^{p}-(1-\theta)(\alpha-k+1)^{p}\right)^{1 / p}}\right) .
$$

Now, subtracting (52) from (51), we arrive at 


$$
\left(\frac{\alpha-k}{\left(\alpha^{p}-(1-\theta)(\alpha-k)^{p}\right)^{1 / p}}\right) f(k+1)=[1-F(k+1)]\left(\frac{\alpha-k}{\left(\alpha^{p}-(1-\theta)(\alpha-k)^{p}\right)^{1 / p}}+\frac{\alpha-k+1}{\left(\alpha^{p}-(1-\theta)(\alpha-k-1)^{p}\right)^{1 / p}}\right),
$$

or

$$
\frac{f(k+1)}{1-F(k+1)}=\frac{(\alpha-k)\left(\alpha^{p}-(1-\theta)(\alpha-k-1)^{p}\right)^{1 / p}}{(\alpha-k+1)\left(\alpha^{p}-(1-\theta)(\alpha-k)^{p}\right)^{1 / p}}-1,
$$

which, in view of (27), implies that $X$ has pmf (26).

Proposition 2.1.8. Let $X: \Omega \rightarrow \mathbb{N}^{*}$ be a random variable. The pmf of $X$ is (29) if and only if

$$
E\left[\left(\frac{1}{(1-\bar{\alpha} \bar{G}(X ; \sigma))}+\frac{1}{(1-\bar{\alpha} \bar{G}(X+1 ; \sigma))}\right) \mid X>k\right]=1-\frac{1}{(1-\bar{\alpha} \bar{G}(k+1 ; \sigma))} .
$$

Proof. If $X$ has pmf (29), then the left-hand side of (53) will be

$$
\begin{aligned}
& (1-F(x))^{-1} \sum_{x=k+1}^{\infty} \frac{\alpha}{\bar{\alpha}}\left(\frac{1}{(1-\bar{\alpha} \bar{G}(k ; \sigma))^{2}}+\frac{1}{(1-\bar{\alpha} \bar{G}(k+1 ; \sigma))^{2}}\right) \\
& =\frac{(1-\bar{\alpha} \bar{G}(k+1 ; \sigma))}{\bar{\alpha} \bar{G}(k+1 ; \sigma)}\left(\frac{1}{(1-\bar{\alpha} \bar{G}(k+1 ; \sigma))^{2}}\right)=1-\frac{1}{(1-\bar{\alpha} \bar{G}(k+1 ; \sigma))} .
\end{aligned}
$$

Conversely, if (53) holds, then

$$
\begin{aligned}
& \sum_{x=k+1}^{\infty}\left\{\left(\frac{1}{(1-\bar{\alpha} \bar{G}(x ; \sigma))}+\frac{1}{(1-\bar{\alpha} \bar{G}(k+1 ; \sigma))}\right) f(x)\right\} \\
& =(1-F(k))\left(1-\frac{1}{(1-\bar{\alpha} \bar{G}(k+1 ; \sigma))}\right)=[(1-F(k+1))+f(k+1)]\left(1-\frac{1}{(1-\bar{\alpha} \bar{G}(k+1 ; \sigma))}\right) .
\end{aligned}
$$

From (53), we also have

$$
\sum_{x=k+2}^{\infty}\left\{\left(\frac{1}{(1-\bar{\alpha} \bar{G}(x ; \sigma))}+\frac{1}{(1-\bar{\alpha} \bar{G}(k+1 ; \sigma))}\right) f(x)\right\}=(1-F(k))\left(1-\frac{1}{(1-\bar{\alpha} \bar{G}(k+2 ; \sigma))}\right)
$$

Now, subtracting (55) from (54), we arrive at

$$
\left(\frac{1}{(1-\bar{\alpha} \bar{G}(k+2 ; \sigma))}-1\right) f(k+1)=(1-F(k+1))\left(\frac{1}{(1-\bar{\alpha} \bar{G}(k+1 ; \sigma))}-\frac{1}{(1-\bar{\alpha} \bar{G}(k+2 ; \sigma))}\right),
$$

or

$$
\frac{f(k+1)}{(1-F(k+1))}=\frac{\bar{G}(k+1 ; \sigma)-\bar{G}(k+2 ; \sigma)}{\bar{G}(k+2 ; \sigma)(1-\bar{\alpha} \bar{G}(k+1 ; \sigma))},
$$

which, in view of (30), implies that $X$ has pmf (29).

\subsection{Characterizations Based on the Hazard Function}

Proposition 2.2.1. Let $X: \Omega \rightarrow \mathbb{N}^{*}$ be a random variable. The pmf of $X$ is (2) if and only if its hazard rate function satisfies the difference equation

$$
h_{F}(k+1)-h_{F}(k)=\frac{B\left(\beta,(k+2)^{-\gamma}+\alpha\right)-B\left(\beta,(k+1)^{-\gamma}+\alpha\right)}{B(\alpha, \beta)-B\left(\beta,(k+2)^{-\gamma}+\alpha\right)}-\frac{B\left(\beta,(k+1)^{-\gamma}+\alpha\right)-B\left(\beta,(k)^{-\gamma}+\alpha\right)}{B(\alpha, \beta)-B\left(\beta,(k+1)^{-\gamma}+\alpha\right)}, k \in \mathbb{N},
$$

with the boundary condition 


$$
h_{F}(1)=\frac{B\left(\beta,(2)^{-\gamma}+\alpha\right)-B(\beta, 1+\alpha)}{B(\alpha, \beta)-B\left(\beta,(2)^{-\gamma}+\alpha\right)} .
$$

Proof. If $X$ has pmf (2), then clearly (56) holds. Now, if (56) holds, then for every $x \in \mathbb{N}$, we have

$$
\begin{aligned}
\sum_{k=1}^{x-1} h_{F}(k+1) & -h_{F}(k)=\sum_{k=1}^{x-1}\left(\frac{B\left(\beta,(k+2)^{-\gamma}+\alpha\right)-B\left(\beta,(x)^{-\gamma}+\alpha\right)}{B(\alpha, \beta)-B\left(\beta,(x+1)^{-\gamma}+\alpha\right)}-\frac{B\left(\beta,(k+1)^{-\gamma}+\alpha\right)-B\left(\beta,(k)^{-\gamma}+\alpha\right)}{B(\alpha, \beta)-B\left(\beta,(k+1)^{-\gamma}+\alpha\right)}\right) \\
= & \sum_{k=1}^{x-1}\left(\frac{B\left(\beta,(x+1)^{-\gamma}+\alpha\right)-B\left(\beta,(k+1)^{-\gamma}+\alpha\right)}{B(\alpha, \beta)-B\left(\beta,(x+1)^{-\gamma}+\alpha\right)}-\frac{B\left(\beta,(2)^{-\gamma}+\alpha\right)-B(\beta, 1+\alpha)}{B(\alpha, \beta)-B\left(\beta,(2)^{-\gamma}+\alpha\right)}\right)
\end{aligned}
$$

or

$$
\sum_{k=1}^{x-1} h_{F}(x)-h_{F}(1)=\sum_{k=1}^{x-1}\left(\frac{B\left(\beta,(x+1)^{-\gamma}+\alpha\right)-B\left(\beta,(k+1)^{-\gamma}+\alpha\right)}{B(\alpha, \beta)-B\left(\beta,(x+1)^{-\gamma}+\alpha\right)}-\frac{B\left(\beta,(2)^{-\gamma}+\alpha\right)-B(\beta, 1+\alpha)}{B(\alpha, \beta)-B\left(\beta,(2)^{-\gamma}+\alpha\right)}\right) .
$$

In view of the fact that $h_{F}(1)=\frac{B\left(\beta,(2)^{-\gamma}+\alpha\right)-B(\beta, 1+\alpha)}{B(\alpha, \beta)-B\left(\beta,(2)^{-\gamma}+\alpha\right)}$, from the last equation we have

$$
h_{F}(x)=\frac{B\left(\beta,(x+1)^{-\gamma}+\alpha\right)-B\left(\beta,(x)^{-\gamma}+\alpha\right)}{B(\alpha, \beta)-B\left(\beta,(x+1)^{-\gamma}+\alpha\right)},
$$

which, in view of (3), implies that $X$ has pmf (2).

Proposition 2.2.2. Let $X: \Omega \rightarrow \mathbb{N}^{*}$ be a random variable. The pmf of $X$ is (6) if and only if its hazard rate function satisfies the difference equation

$$
h_{F}(k+1)-h_{F}(k)=\frac{1}{q}\left\{\left(\frac{k+2}{k+1}\right)^{\alpha}-\left(\frac{k+3}{k+2}\right)^{\alpha}\right\}, \quad k \in \mathbb{N},
$$

with the boundary condition $h_{F}(0)=1-\frac{2^{\alpha}}{q}$.

Proof. The proof is similar to that of Proposition 2.2.1. We, however, give the proof for the sake of completeness. If $X$ has pmf (6), then clearly (57) holds. Now, if (57) holds, then for every $x \in \mathbb{N}$, we have

$$
\sum_{k=0}^{x-1} h_{F}(k+1)-h_{F}(k)=\sum_{k=0}^{x-1} \frac{1}{q}\left\{\left(\frac{k+2}{k+1}\right)^{\alpha}-\left(\frac{k+3}{k+2}\right)^{\alpha}\right\}=\frac{2^{\alpha}}{q}-\frac{1}{q}\left(\frac{x+2}{x+1}\right)^{\alpha}
$$

or

$$
h_{F}(x)-h_{F}(0)=\frac{2^{\alpha}}{q}-\frac{1}{q}\left(\frac{x+2}{x+1}\right)^{\alpha}
$$

In view of the fact that $h_{F}(0)=1-\frac{2^{\alpha}}{q}$, from the last equation we have

$$
h_{F}(x)=1-\frac{1}{q}\left(\frac{x+2}{x+1}\right)^{\alpha}
$$

which, in view of (7), implies that $X$ has pmf (6).

Proposition 2.2.3. Let $X: \Omega \rightarrow \mathbb{N}^{*}$ be a random variable. The pmf of $X$ is (10) if and only if its hazard rate function satisfies the difference equation 


$$
h_{F}(k+1)-h_{F}(k)=\frac{1}{p}\left\{\frac{(1-p)^{2}(1+\beta(k+1))}{(1-p)(1+\beta(k+1))+\beta}-\frac{(1-p)^{2}(1+\beta k)}{(1-p)(1+\beta k)+\beta}\right\}, \quad x \in \mathbb{N}^{*},
$$

with the boundary condition $h_{F}(0)=\frac{(1-p)^{2}}{p(1-p+\beta)}$.

$$
\sum_{k=1}^{x-1} h_{F}(k+1)-h_{F}(k)=\frac{(1-p)^{2}}{p} \sum_{k=1}^{x-1}\left\{\frac{(1+\beta(k+1))}{(1-p)(1+\beta(k+1))+\beta}-\frac{(1+\beta k)}{(1-p)(1+\beta k)+\beta}\right\},
$$

or

$$
h_{F}(x)-h_{F}(0)=\frac{(1-p)^{2}}{p} \sum_{k=1}^{x-1}\left\{\frac{(1+\beta x)}{(1-p)(1+\beta x)+\beta}-\frac{1}{1-p+\beta}\right\} .
$$

In view of the fact that $h_{F}(0)=\frac{(1-p)^{2}}{p(1-p+\beta)}$, from the last equation we have

$$
h_{F}(x)=\frac{(1-p)^{2}(1+\beta x)}{p[(1-p)(1+\beta x)+\beta]},
$$

which, in view of (11), implies that $X$ has pmf (10).

Proposition 2.2.4. Let $X: \Omega \rightarrow \mathbb{N}^{*}$ be a random variable. The pmf of $X$ is (14) for $\alpha=1$, if and only if its hazard rate function satisfies the difference equation

$$
h_{F}(k+1)-h_{F}(k)=\theta^{2}\left\{\frac{1+(k+1)}{\theta(2+(k+1))+1}-\frac{1+k}{\theta(2+k)+1}\right\}, \quad k \in \mathbb{N}^{*} \text {, }
$$

with the boundary condition $h_{F}(0)=\frac{\theta^{2}}{2 \theta+1}$.

Proof. If $X$ has pmf (14), for $=0$, then clearly (59) holds. Now, if (59) holds, then for every $x \in \mathbb{N}$, we have

$$
\begin{gathered}
\sum_{k=1}^{x-1} h_{F}(k+1)-h_{F}(k)=\theta^{2} \sum_{k=1}^{x-1}\left\{\frac{1+(k+1)}{\theta(2+(k+1))+1}-\frac{1+k}{\theta(2+k)+1}\right\}, \\
h_{F}(x)-h_{F}(0)=\theta^{2}\left\{\frac{1+x}{\theta(2+x)+1}-\frac{1}{2 \theta+1}\right\},
\end{gathered}
$$

In view of the fact that $h_{F}(0)=\frac{\theta^{2}}{2 \theta+1}$, from the last equation we have

$$
h_{F}(x)=\frac{\theta^{2}(1+x)}{\theta(2+x)+1}
$$

which, in view of (15), implies that $X$ has $\operatorname{pmf}(14)$ for $\alpha=1$.

Proposition 2.2.5. Let $X: \Omega \rightarrow \mathbb{N}^{*}$ be a random variable. The pmf of $X$ is (18) if and only if its hazard rate function satisfies the difference equation

$$
h_{F}(k+1)-h_{F}(k)=\frac{(1-\theta)\left(1-p_{1} \theta\right)}{\theta}\left[\frac{1-p_{1}^{k+1}}{\left(1-p_{1} \theta\right)-(1-\theta) p_{1}^{k+2}}-\frac{1-p_{1}^{k}}{\left(1-p_{1} \theta\right)-(1-\theta) p_{1}^{k+1}}\right] \text {, }
$$

$k \in \mathbb{N}$, with the boundary condition $h_{F}(1)=\frac{1}{1+(1-\theta) p_{1}}-\theta$

Proof. If $X$ has pmf (18), then clearly (60) holds. Now, if (60) holds, then for every $x \in \mathbb{N}$, we have 


$$
\begin{gathered}
\sum_{k=1}^{x-1} h_{F}(k+1)-h_{F}(k)=\frac{(1-\theta)\left(1-p_{1} \theta\right)}{\theta} \sum_{k=1}^{x-1}\left[\frac{1-p_{1}^{k+1}}{\left(1-p_{1} \theta\right)-(1-\theta) p_{1}^{k+2}}-\frac{1-p_{1}^{k}}{\left(1-p_{1} \theta\right)-(1-\theta) p_{1}^{k+1}}\right], \\
=\frac{(1-\theta)\left(1-p_{1} \theta\right)}{\theta} \sum_{k=1}^{x-1}\left[\frac{1-p_{1}^{x}}{\left(1-p_{1} \theta\right)-(1-\theta) p_{1}^{x+1}}-\frac{1-p_{1}}{\left(1-p_{1} \theta\right)-(1-\theta) p_{1}^{2}}\right],
\end{gathered}
$$

or

$$
h_{F}(x)-h_{F}(1)=\frac{(1-\theta)\left(1-p_{1} \theta\right)}{\theta}\left[\frac{1-p_{1}^{x}}{\left(1-p_{1} \theta\right)-(1-\theta) p_{1}^{x+1}}-\frac{1-p_{1}}{\left(1-p_{1} \theta\right)-(1-\theta) p_{1}^{2}}\right] \text {, }
$$

In view of the fact that $h_{F}(1)=\frac{1}{1+(1-\theta) p_{1}}-\theta$. from the last equation we have

$$
h_{F}(x)=\frac{(1-\theta)\left(1-p_{1} \theta\right)\left(1-p_{1}^{x}\right)}{\theta\left[\left(1-p_{1} \theta\right)-(1-\theta) p_{1}^{x+1}\right]},
$$

which, in view of (19), implies that $X$ has pmf (18).

Proposition 2.2.6. Let $X: \Omega \rightarrow \mathbb{N}^{*}$ be a random variable. The pmf of $X$ is (22) if and only if its hazard rate function satisfies the difference equation

$$
h_{F}(k+1)-h_{F}(k)=\frac{G^{\alpha}(k+2)-G^{\alpha}(k+1)}{\left(\theta+\bar{\theta} G^{\alpha}(k+1)\right)\left(1-G^{\alpha}(k+2)\right)}-\frac{G^{\alpha}(k+1)-G^{\alpha}(k)}{\left(\theta+\bar{\theta} G^{\alpha}(k)\right)\left(1-G^{\alpha}(k+1)\right)},
$$

$k \in \mathbb{N}^{*}$, with the boundary condition $h_{F}(0)=\frac{G^{\alpha}(1)}{\theta\left(1-G^{\alpha}(1)\right)}$.

Proof. If $X$ has pmf (22), then clearly (61) holds. Now, if (61) holds, then for every $x \in \mathbb{N}$, we have

$$
\begin{aligned}
\sum_{k=1}^{x-1} h_{F}(k+1)-h_{F}(k)= & \sum_{k=1}^{x-1} \frac{G^{\alpha}(k+2)-G^{\alpha}(k+1)}{\left(\theta+\bar{\theta} G^{\alpha}(k+1)\right)\left(1-G^{\alpha}(k+2)\right)}-\frac{G^{\alpha}(k+1)-G^{\alpha}(k)}{\left(\theta+\bar{\theta} G^{\alpha}(k)\right)\left(1-G^{\alpha}(k+1)\right)}, \\
& =\frac{G^{\alpha}(x+1)-G^{\alpha}(x)}{\left(\theta+\bar{\theta} G^{\alpha}(x)\right)\left(1-G^{\alpha}(x+1)\right)}-\frac{G^{\alpha}(1)}{\theta\left(1-G^{\alpha}(1)\right)},
\end{aligned}
$$

or

$$
h_{F}(x)-h_{F}(0)=\frac{G^{\alpha}(x+1)-G^{\alpha}(x)}{\left(\theta+\bar{\theta} G^{\alpha}(x)\right)\left(1-G^{\alpha}(x+1)\right)}-\frac{G^{\alpha}(1)}{\theta\left(1-G^{\alpha}(1)\right)},
$$

In view of the fact that $h_{F}(0)=\frac{G^{\alpha}(1)}{\theta\left(1-G^{\alpha}(1)\right)}$, from the last equation we have

$$
h_{F}(x)=\frac{G^{\alpha}(x+1)-G^{\alpha}(x)}{\left(\theta+\bar{\theta} G^{\alpha}(x)\right)\left(1-G^{\alpha}(x+1)\right)},
$$

which, in view of (23), implies that $X$ has pmf (22).

Proposition 2.2.7. Let $X: \Omega \rightarrow I \backslash\{0\}$ be a random variable. The pmf of $X$ is (26) if and only if its hazard rate function satifies the difference equation

$$
h_{F}(k+1)-h_{F}(k)=\frac{(\alpha-k)\left(\alpha^{p}-(1-\theta)(\alpha-k-1)^{p}\right)^{1 / p}}{(\alpha-k-1)\left(\alpha^{p}-(1-\theta)(\alpha-k)^{p}\right)^{1 / p}}-\frac{(\alpha-k+1)\left(\alpha^{p}-(1-\theta)(\alpha-k)^{p}\right)^{1 / p}}{(\alpha-k)\left(\alpha^{p}-(1-\theta)(\alpha-k+1)^{p}\right)^{1 / p}},
$$

$X \in I \backslash\{0\}$, with the boundary condition $h_{F}(1)=\frac{\left[\alpha^{p}-(1-\theta)\left(\alpha^{p}-1^{p}\right)\right]^{1 / p}}{(\alpha-1) \theta^{1 / p}}-1$.

Proof. If X has pmf (26), then clearly (62) holds. Now, if (62) holds, then for every $x \in I \backslash\{0\}$, we have 


$$
\begin{aligned}
\sum_{k=1}^{x-1} h_{F}(k+1)- & h_{F}(k)=\sum_{k=1}^{x-1} \frac{(\alpha-k)\left(\alpha^{p}-(1-\theta)(\alpha-k-1)^{p}\right)^{1 / p}}{(\alpha-k-1)\left(\alpha^{p}-(1-\theta)(\alpha-k)^{p}\right)^{1 / p}}-\frac{(\alpha-k+1)\left(\alpha^{p}-(1-\theta)(\alpha-k)^{p}\right)^{1 / p}}{(\alpha-k)\left(\alpha^{p}-(1-\theta)(\alpha-k+1)^{p}\right)^{1 / p}}, \\
= & \frac{(\alpha-x+1)\left(\alpha^{p}-(1-\theta)(\alpha-x)^{p}\right)^{1 / p}}{(\alpha-k)\left(\alpha^{p}-(1-\theta)(\alpha-x+1)^{p}\right)^{1 / p}}-\frac{\left(\alpha^{p}-(1-\theta)(\alpha-1)^{p}\right)^{1 / p}}{(\alpha-1) \theta^{1 / p}},
\end{aligned}
$$

or

$$
h_{F}(x)-h_{F}(0)=\frac{(\alpha-x+1)\left(\alpha^{p}-(1-\theta)(\alpha-x)^{p}\right)^{1 / p}}{(\alpha-k)\left(\alpha^{p}-(1-\theta)(\alpha-x+1)^{p}\right)^{1 / p}}-\frac{\left(\alpha^{p}-(1-\theta)(\alpha-1)^{p}\right)^{1 / p}}{(\alpha-1) \theta^{1 / p}} .
$$

In view of the fact that $h_{F}(1)=\frac{\left(\alpha^{p}-(1-\theta)(\alpha-1)^{p}\right)^{1 / p}}{(\alpha-1) \theta^{1 / p}}-1$, from the last equation we have

$$
h_{F}(x)=\frac{(\alpha-x+1)\left(\alpha^{p}-(1-\theta)(\alpha-x)^{p}\right)^{1 / p}}{(\alpha-k)\left(\alpha^{p}-(1-\theta)(\alpha-x+1)^{p}\right)^{1 / p}} .
$$

which, in view of (27), implies that $X$ has pmf (26).

Proposition 2.1.8. Let $X: \Omega \rightarrow \mathbb{N}^{*}$ be a random variable. The pmf of $X$ is (29) if and only if its hazard rate function satisfies the difference equation

$$
h_{F}(k+1)-h_{F}(k)=\frac{\bar{G}(k+1 ; \sigma)-\bar{G}(k+2 ; \sigma)}{\bar{G}(k+1 ; \sigma)(1-\bar{\alpha} \bar{G}(k+1 ; \sigma))}-\frac{\bar{G}(k ; \sigma)-\bar{G}(k+1 ; \sigma)}{\bar{G}(k+1 ; \sigma)(1-\bar{\alpha} \bar{G}(k ; \sigma))},
$$

$k \in \mathbb{N}^{*}$, with the boundary condition $h_{F}(0)=\frac{\bar{G}(0)-G(1 ; \sigma)}{\bar{G}(1 ; \sigma)(1-\bar{\alpha} \bar{G}(0 ; \sigma))}$.

Proof. If X has pmf (29), then clearly (63) holds. Now, if (63) holds, then for every $x \in \mathbb{N}^{*}$, we have

$$
\begin{gathered}
\sum_{k=1}^{x-1} h_{F}(k+1)-h_{F}(k)=\sum_{k=1}^{x-1} \frac{\bar{G}(k+1 ; \sigma)-\bar{G}(k+2 ; \sigma)}{\bar{G}(k+2 ; \sigma)(1-\bar{\alpha} \bar{G}(k+1 ; \sigma))}-\frac{\bar{G}(k ; \sigma)-\bar{G}(k+1 ; \sigma)}{\bar{G}(k+1 ; \sigma)(1-\bar{\alpha} \bar{G}(k ; \sigma))}, \\
=\frac{\bar{G}(x ; \sigma)-\bar{G}(x+1 ; \sigma)}{\bar{G}(x+1 ; \sigma)(1-\bar{\alpha} \bar{G}(k ; \sigma))}-\frac{\bar{G}(0 ; \sigma)-\bar{G}(1 ; \sigma)}{\bar{G}(1 ; \sigma)(1-\bar{\alpha} \bar{G}(k ; \sigma))}, h_{F}(x)-h_{F}(0)=\frac{\bar{G}(x ; \sigma)-\bar{G}(x+1 ; \sigma)}{\bar{G}(k+2 ; \sigma)(1-\bar{\alpha} \bar{G}(k+1 ; \sigma))}-\frac{\bar{G}(0)-\bar{G}(1 ; \sigma)}{\bar{G}(1 ; \sigma)(1-\bar{\alpha} \bar{G}(k ; \sigma))} .
\end{gathered}
$$

In view of the fact that $h_{F}(0)=\frac{\bar{G}(0)-G(1 ; \sigma)}{\bar{G}(1 ; \sigma)(1-\bar{\alpha} \bar{G}(0 ; \sigma))}$, from the last equation we have

$$
h_{F}(x)=\frac{\bar{G}(x ; \sigma)-\bar{G}(x+1 ; \sigma)}{\bar{G}(x+1 ; \sigma)(1-\bar{\alpha} \bar{G}(x ; \sigma))},
$$

which, in view of (30), implies that $X$ has pmf (29).

\subsection{Characterizations Based on the Reverse Hazard Function}

Proposition 2.3.1. Let $X: \Omega \rightarrow \mathbb{N}^{*}$ be a random variable. The pmf of $X$ is (2) if and only if its reverse hazard rate function satisfies the difference equation

$$
r_{F}(k+1)-r_{F}(k)=\frac{B\left(\beta,(k)^{-\gamma}+\alpha\right)}{B\left(\beta,(k+1)^{-\gamma}+\alpha\right)}-\frac{B\left(\beta,(k+1)^{-\gamma}+\alpha\right)}{B\left(\beta,(k+2)^{-\gamma}+\alpha\right)}, \quad k \in \mathbb{N},
$$

with the boundary condition $r_{F}(1)=1-\frac{B(\beta, 1+\alpha)}{B\left(\beta,(2)^{-\gamma}+\alpha\right)}$. 
Proof. If X has pmf (2), then clearly (64) holds. Now, if (64) holds, then for every $x \in \mathbb{N}$, we have

$$
\sum_{k=1}^{x-1} r_{F}(k+1)-r_{F}(k)=\sum_{k=1}^{x-1} \frac{B\left(\beta,(k)^{-\gamma}+\alpha\right)}{B\left(\beta,(k+1)^{-\gamma}+\alpha\right)}-\frac{B\left(\beta,(k+1)^{-\gamma}+\alpha\right)}{B\left(\beta,(k+2)^{-\gamma}+\alpha\right)},
$$

or

$$
r_{F}(x)-r_{F}(1)=\sum_{k=1}^{x-1} \frac{B(\beta, 1+\alpha)}{B\left(\beta,(2)^{-\gamma}+\alpha\right)}-\frac{B\left(\beta,(x)^{-\gamma}+\alpha\right)}{B\left(\beta,(x+1)^{-\gamma}+\alpha\right)},
$$

In view of the fact that ${ }^{r_{F}}(1)=1-\frac{B(\beta, 1+\alpha)}{B\left(\beta,(2)^{-\gamma}+\alpha\right)}$ from the last equation we have

$$
r_{F}(x)=1-\frac{B\left(\beta,(x)^{-\gamma}+\alpha\right)}{B\left(\beta,(x+1)^{-\gamma}+\alpha\right)},
$$

which, in view of (4), implies that $X$ has pmf (2).

Proposition 2.3.2. Let $X: \Omega \rightarrow \mathbb{N}^{*}$ be a random variable. The pmf of $X$ is (4) if and only if its reverse hazard rate function satisfies the difference equation

$$
r_{F}(k+1)-r_{F}(k)=\frac{1-\frac{q^{k}}{(k+1)^{\alpha}}}{1-\frac{q^{k+1}}{(k+2)^{\alpha}}}-\frac{1-\frac{q^{k+1}}{(k+2)^{\alpha}}}{1-\frac{q^{k+2}}{(k+3)^{\alpha}}}, \quad k \in \mathbb{N},
$$

with the boundary condition $r_{F}(0)=1$.

Proof. The proof is similar to that of Proposition 2.3.1. We, however, give the proof for the sake of completeness. If $X$ has pmf (6), then clearly (65) holds. Now, if (65) holds, then for every $x \in \mathbb{N}$, we have

$$
\sum_{k=0}^{x-1} r_{F}(k+1)-r_{F}(k)=\sum_{k=0}^{x-1} \frac{1-\frac{q^{k}}{(k+1)^{\alpha}}}{1-\frac{q^{k+1}}{(k+2)^{\alpha}}}-\frac{1-\frac{q^{k+1}}{(k+2)^{\alpha}}}{1-\frac{q^{k+2}}{(k+3)^{\alpha}}},
$$

or

$$
r_{F}(x)-r_{F}(0)=\frac{1-\frac{q^{x}}{(x+1)^{\alpha}}}{1-\frac{q^{x+1}}{(x+2)^{\alpha}}}
$$

In view of the fact that $h_{F}(0)=1$, from the last equation we have

which, in view of (8), implies that $X$ has pmf (6).

$$
r_{F}(x)=1-\frac{1-\frac{q^{x}}{(x+1)^{\alpha}}}{1-\frac{q^{x+1}}{(x+2)^{\alpha}}} .
$$

Proposition 2.3.3. Let $\mathrm{X}$ : ! N be a random variable. The pmf of $X$ is (10) if and only if its reverse hazard rate function satisfies the difference equation

$$
r_{F}(k+1)-r_{F}(k)=\frac{(1-p)^{2}(1+\beta(k+1)) p^{k+1}}{1+p(\beta-1)-p[(1-p)(1+\beta(k+1))+\beta] p^{k+2}}-\frac{(1-p)^{2}(1+\beta k) p^{k}}{1+p(\beta-1)-p[(1-p)(1+\beta k)+\beta] p^{k+1}},
$$

$k \in \mathbb{N}^{*}$, with the boundary condition $r_{F}(0)=1$.

Proof. If $X$ has pmf (10), then clearly (66) holds. Now, if (66) holds, then for every $x \in \mathbb{N}$, we have 


$$
\sum_{k=1}^{x-1} r_{F}(k+1)-r_{F}(k)=\sum_{k=1}^{x-1}\left(\frac{(1-p)^{2}(1+\beta(k+1)) p^{k+1}}{1+p(\beta-1)-p[(1-p)(1+\beta(k+1))+\beta] p^{k+2}}-\frac{(1-p)^{2}(1+\beta k) p^{k}}{1+p(\beta-1)-p[(1-p)(1+\beta k)+\beta] p^{k+1}}\right),
$$

or

$$
r_{F}(x)-r_{F}(0)=\frac{(1-p)^{2}(1+\beta x) p^{x}}{1+p(\beta-1)-p[(1-p)(1+\beta x)+\beta] p^{x+1}}-1,
$$

In view of the fact that $r_{F}(0)=1$, from the last equation we have

$$
h_{F}(x)=\frac{(1-p)^{2}(1+\beta x)}{p[(1-p)(1+\beta x)+\beta]},
$$

which, in view of (12), implies that $X$ has pmf (10).

Proposition 2.3.4. Let $X: \Omega \rightarrow \mathbb{N}^{*}$ be a random variable. The pmf of $X$ is (14) if and only if its reverse hazard rate function satisfies the difference equation

$$
r_{F}(k+1)-r_{F}(k)=\frac{1+(2+k)}{(1+\theta)^{3}-(\theta(3+k)+1)}-\frac{\theta^{2}(1+k)}{(1+\theta)^{k+2}-(\theta(2+k)+1)},
$$

$k \in \mathbb{N}^{*}$ with the boundary condition $r_{F}(0)=1$.

Proof. If $X$ has pmf (14), for $=0$, then clearly (67) holds. Now, if (67) holds, then for every $x \in \mathbb{N}$, we have

$$
\sum_{k=1}^{x-1} r_{F}(k+1)-r_{F}(k)=\sum_{k=1}^{x-1}\left\{\frac{\theta^{2}(2+k)}{(1+\theta)^{k+3}-(\theta(3+k)+1)}-\frac{\theta^{2}(1+k)}{(1+\theta)^{k+2}-(\theta(2+k)+1)}\right\},
$$

or

$$
r_{F}(x)-r_{F}(0)=\frac{\theta^{2}(1+x)}{(1+\theta)^{x+2}-(\theta(2+x)+1)}-1 .
$$

In view of the fact that $r_{F}(0)=1$, from the last equation we have

$$
r_{F}(x)=\frac{\theta^{2}(1+x)}{(1+\theta)^{x+2}-\theta(2+x)+1},
$$

which, in view of (16), implies that $X$ has pmf (14).

Proposition 2.3.5. Let $X: \Omega \rightarrow \mathbb{N}^{*}$ be a random variable. The pmf of $X$ is (18) if and only if its reverse hazard rate function satisfies the difference equation

$$
r_{F}(k+1)-r_{F}(k)=\left[\frac{(1-\theta)\left(1-p_{1} \theta\right)\left(1-p_{1}^{k+1}\right) \theta^{k}}{\left(1-p_{1}\right)-\theta^{k}\left[\left(1-p_{1} \theta\right)-(1-\theta) p_{1}^{k+2}\right]}-\frac{(1-\theta)\left(1-p_{1} \theta\right)\left(1-p_{1}^{k}\right) \theta^{k-1}}{\left(1-p_{1}\right)-\theta^{k}\left[\left(1-p_{1} \theta\right)-(1-\theta) p_{1}^{k+1}\right]}\right],
$$

$k \in \mathbb{N}$, with the boundary condition $r_{F}(1)=\frac{(1-\theta)\left(1-p_{1} \theta\right)\left(1-p_{1}\right)}{\left(1-p_{1}\right)-\theta\left[\left(1-p_{1} \theta\right)-(1-\theta) p_{1}^{2}\right]}$.

Proof. If $X$ has pmf (18), then clearly (68) holds. Now, if (68) holds, then for every $x \in \mathbb{N}$, we have

$$
\sum_{k=1}^{x-1} r_{F}(k+1)-r_{F}(k)=\sum_{k=1}^{x-1}\left[\frac{(1-\theta)\left(1-p_{1} \theta\right)\left(1-p_{1}^{k+1}\right) \theta^{k}}{\left(1-p_{1}\right)-\theta^{k}\left[\left(1-p_{1} \theta\right)-(1-\theta) p_{1}^{k+2}\right]}-\frac{(1-\theta)\left(1-p_{1} \theta\right)\left(1-p_{1}^{k}\right) \theta^{k-1}}{\left(1-p_{1}\right)-\theta^{k}\left[\left(1-p_{1} \theta\right)-(1-\theta) p_{1}^{k+1}\right]}\right],
$$

or

$$
r_{F}(x)-r_{F}(1)=\left[\frac{(1-\theta)\left(1-p_{1} \theta\right)\left(1-p_{1}^{x}\right) \theta^{x-1}}{\left(1-p_{1}\right)-\theta^{x}\left[\left(1-p_{1} \theta\right)-(1-\theta) p_{1}^{x+1}\right]}-\frac{(1-\theta)\left(1-p_{1} \theta\right)\left(1-p_{1}\right)}{\left(1-p_{1}\right)-\theta\left[\left(1-p_{1} \theta\right)-(1-\theta) p_{1}^{2}\right]}\right] .
$$


In view of the fact that $r_{F}(1)=\frac{(1-\theta)\left(1-p_{1} \theta\right)\left(1-p_{1}\right)}{\left(1-p_{1}\right)-\theta\left[\left(1-p_{1} \theta\right)-(1-\theta) p_{1}^{2}\right]}$, from the last equation we have

$$
r_{F}(x)=\frac{(1-\theta)\left(1-p_{1} \theta\right)\left(1-p_{1}^{x}\right) \theta^{x-1}}{\left(1-p_{1}\right)-\theta^{x}\left[\left(1-p_{1} \theta\right)-(1-\theta) p_{1}^{x+1}\right]},
$$

which, in view of (20), implies that $X$ has pmf (18).

Proposition 2.3.6. Let $X: \Omega \rightarrow \mathbb{N}^{*}$ be a random variable. The pmf of $X$ is (22) if and only if its reverse hazard rate function satisfies the difference equation

$$
r_{F}(k+1)-r_{F}(k)=\frac{\theta\left(G^{\alpha}(k+2)-G^{\alpha}(k+1)\right)}{G^{\alpha}(k+2)\left(\theta+\bar{\theta} G^{\alpha}(k+1)\right)}-\frac{\theta\left(G^{\alpha}(k+1)-G^{\alpha}(k)\right)}{G^{\alpha}(k+1)\left(\theta+\bar{\theta} G^{\alpha}(k)\right)},
$$

$k \in \mathbb{N}^{*}$, with the boundary condition $r_{F}(0)=1$.

Proof. If $X$ has pmf (22), then clearly (69) holds. Now, if (69) holds, then for every $x \in \mathbb{N}$, we have

$$
\sum_{k=1}^{x-1} r_{F}(k+1)-r_{F}(k)=\sum_{k=1}^{x-1} \frac{\theta\left(G^{\alpha}(k+2)-G^{\alpha}(k+1)\right)}{G^{\alpha}(k+2)\left(\theta+\bar{\theta} G^{\alpha}(k+1)\right)}-\frac{\theta\left(G^{\alpha}(k+1)-G^{\alpha}(k)\right)}{G^{\alpha}(k+1)\left(\theta+\bar{\theta} G^{\alpha}(k)\right)},
$$

or

$$
r_{F}(x)-r_{F}(0)=\frac{\theta\left(G^{\alpha}(x+1)-G^{\alpha}(x)\right)}{G^{\alpha}(x+1)\left(\theta+\bar{\theta} G^{\alpha}(x)\right)}-1 .
$$

In view of the fact that $r_{F}(0)=1$, from the last equation we have

$$
r_{F}(x)=\frac{\theta\left(G^{\alpha}(x+1)-G^{\alpha}(x)\right)}{G^{\alpha}(x+1)\left(\theta+\bar{\theta} G^{\alpha}(x)\right)},
$$

which, in view of (24), implies that $X$ has pmf (22).

Proposition 2.3.7. Let $X: \Omega \rightarrow \mathbb{N}^{*}$ be a random variable. The pmf of $X$ is (29) if and only if its reverse hazard rate function satifies the difference equation

$$
r_{F}(k+1)-r_{F}(k)=\frac{\bar{G}(k+1 ; \sigma)-\bar{G}(k+2 ; \sigma)}{(1-\bar{G}(k+2 ; \sigma))-(1-\bar{\alpha} \bar{G}(k+1 ; \sigma))}-\frac{\bar{G}(k ; \sigma)-\bar{G}(k+1 ; \sigma)}{(1-\bar{G}(k+1 ; \sigma))-(1-\bar{\alpha} \bar{G}(k ; \sigma))},
$$

$k \in \mathbb{N}$, with the boundary condition $r_{F}(0)=\frac{\bar{G}(0 ; \sigma)-\bar{G}(1 ; \sigma)}{\bar{G}(1 ; \sigma)-(1-\bar{\alpha} \bar{G}(0 ; \sigma))}$.

Proof. If $X$ has pmf (22), then clearly (70) holds. Now, if (70) holds, then for every $x \in \mathbb{N}$, we have

$$
\sum_{k=1}^{x-1} r_{F}(k+1)-r_{F}(k)=\sum_{k=1}^{x-1} \alpha\left\{\frac{\bar{G}(k+1 ; \sigma)-\bar{G}(k+2 ; \sigma)}{(1-\bar{G}(k+2 ; \sigma))-(1-\bar{\alpha} \bar{G}(k+1 ; \sigma))}-\frac{\bar{G}(k ; \sigma)-\bar{G}(k+1 ; \sigma)}{(1-\bar{G}(k+1 ; \sigma))-(1-\bar{\alpha} \bar{G}(k ; \sigma))}\right\},
$$

or

$$
r_{F}(x)-r_{F}(0)=\alpha\left\{\frac{\bar{G}(x ; \sigma)-\bar{G}(x+1 ; \sigma)}{(1-\bar{G}(x+1 ; \sigma))-(1-\bar{\alpha} \bar{G}(x ; \sigma))}-\frac{\bar{G}(0 ; \sigma)-\bar{G}(1 ; \sigma)}{(1-\bar{G}(1 ; \sigma))-(1-\bar{\alpha} \bar{G}(0 ; \sigma))}\right\} .
$$

In view of the fact that $r_{F}(0)=\frac{\bar{G}(0 ; \sigma)-\bar{G}(1 ; \sigma)}{\bar{G}(1 ; \sigma)-(1-\bar{\alpha} \bar{G}(0 ; \sigma))}$, from the last equation we have

$$
r_{F}(x)=\alpha\left(\frac{\bar{G}(x ; \sigma)-\bar{G}(x+1 ; \sigma)}{(1-\bar{\alpha} \bar{G}(x+1 ; \sigma))-(1-\bar{\alpha} \bar{G}(x ; \sigma))}\right) .
$$

which, in view of (31), implies that $X$ has pmf (29).

Remark 2. Para and Jan presented five sub-models of DIWB distribution resulted from taking selected parameter values. 
We list below these sub-models. We believe that the pmf formulas for three of them are not complete as was the case with that of DIWB mentioned in Remarks 1 (a).

(A) For $\gamma=1$, the DIWB reduces to, what the authors called, a compound of Inverse Geometric (IG) with Beta (B), which has the following $\mathrm{pmf}$

$$
f(x ; \alpha, \beta, 1)=\left\{\begin{array}{cc}
\frac{\alpha+1}{\alpha+\beta+1}, & x=0, \\
B\left(\beta,(x+1)^{-1}+\alpha\right)-B\left(\beta,(x)^{-1}+\alpha\right), & x \in \mathbb{N}
\end{array} .\right.
$$

(B) For $\alpha=\beta=1$, the DIWB reduces to, what the authors called, a compound of Discrete Inverse Weibull (DIW) with Uniform (U), which has the following pmf

$$
f(x ; 1,1, \gamma)=\left\{\begin{array}{cc}
\frac{2}{3}, & x=0, \\
B\left(1,(x+1)^{-\gamma}+1\right)-B\left(1,(x)^{-\gamma}+1\right), & x \in \mathbb{N}
\end{array}\right.
$$

(C) For $\alpha=\beta=\gamma=1$, the DIWB reduces to, what the authors called, a compound of Inverse Geometric (IG) with Uniform (U), which has the following pmf

$$
f(x ; 1,1,1)=\frac{1}{(x+1)(x+2)} .
$$

(D) For $\alpha=\beta=\gamma=1$, the DIWB reduces to, what the authors called, a compound of Inverse Rayleigh (IR) with Uniform $(\mathrm{U})$, which has the following pmf

$$
f(x ; 1,1,2)=\frac{2 x+1}{\left(x^{2}+1\right)\left(\left(x^{2}+1\right)+1\right)} .
$$

(E) For $\gamma=2$, the DIWB reduces to, what the authors called, a compound of Discrete Rayleigh (DR) with Beta (B), which has the following pmf

$$
f(x ; \alpha, \beta, 2)=\left\{\begin{array}{cc}
\frac{\alpha+1}{\alpha+\beta+1}, & x=0, \\
B\left(\beta,(x+1)^{-2}+\alpha\right)-B\left(\beta,(x)^{-2}+\alpha\right), & x \in \mathbb{N}
\end{array} .\right.
$$

Remark 3. Clearly, much simpler characterizations, similar to the Propositions 2.1.1 and 2.2.1, can be stated for the above mentioned sub-models of DIWB.

\section{References}

Bhati, D., \& Bakouch, H. S. (2018). A new in ifnitely divisible discrete distribution with applications to count data modeling, Communications in Statistics: Theory and Methods. (Forthcoming). https://doi.org/10.1080/03610926.2018.1433847

Grine, R., \& Zeghdoudi, H. (2017). On Poisson quasi Lindley distribution and its applications, J. of Modern Applied Statistical Methods, 16(2), 403-417. https://doi.org/10.22237/jmasm/1509495660

Hussain, T., Aslam, M., \& Ahmad, M. (2016). A two parameter discrete Lindley distribution, Revista Colombiana de Estadistica, 39(1), 45-61. https://doi.org/10.15446/rce.v39n1.55138

Jayakumar, K., \& Sankaran, K. K. (2018). A generalization of discrete Weibull distribution. Commun. in Statist-Theory and Methods, 47(24), 6064-6078. https://doi.org/10.1080/03610926.2017.1406115

Kumar, C. S., \& Sreejakumari, S. (2016). Extended inverted geometric distribution, International J. of Statistical Distributions and Applications, 2(1), 8-13. https://doi.org/10.11648/j.ijsd.20160201.12

Para, B. A., \& Jan, T. R. (2018). Discrete inverse Weibull beta model: properties and applications in health science, Pak. J. Statist., 34(3), 229-249.

Prasanth, C. B., \& Sandhya, E. (2016). A generalized discrete uniform distribution, J. Stat. Appl. Pro., 5(1), $109-121$. https://doi.org/10.18576/jsap/030110

Supanekar, S. R., \& Shirke, D. T. (2015). A new discrete family of distributions, ProbStat Forum, 8, 83-94. 


\section{Copyrights}

Copyright for this article is retained by the author(s), with first publication rights granted to the journal.

This is an open-access article distributed under the terms and conditions of the Creative Commons Attribution license (http://creativecommons.org/licenses/by/4.0/). 